\title{
Oral Squamous Cell Carcinoma of Palate - A Case Report and Review of Literature
}

\author{
Laliytha Kumar Bijai,", Philips Mathew ${ }^{2}$, Venkatesh Jayaraman², Ravi David Austin ${ }^{2}$ \\ ${ }^{1}$ Department of Dentistry, Sree Balaji Medical College and Hospital, Bharath University, Chennai, India \\ ${ }^{2}$ Department of Oral Medicine and Radiology, Rajah Muthiah Dental College, Annamalai University, Chidambaram, India \\ *Corresponding author: lalithadentist@gmail.com
}

Received August 01, 2014; Revised August 25, 2014; Accepted August 28, 2014

\begin{abstract}
Oral squamous cell carcinoma (OSCC) is a significant public health problem worldwide. More than 95\% of the carcinomas of the oral cavity are of squamous cell type, in nature. The development of OSCC is a multistep process requiring the accumulation of multiple genetic alterations, influenced by environmental influences, including tobacco products, alcohol consmption, viral infection, chronic inflammation and also patient's genetic predisposition. Here we present a case of oral squamous cell carcinoma of palate in a 62-year-old male patient.
\end{abstract}

Keywords: oral squamous cell carcinoma, betel quid, carcinogenesis

Cite This Article: Laliytha Kumar Bijai, Philips Mathew, Venkatesh Jayaraman, and Ravi David Austin, "Oral Squamous Cell Carcinoma of Palate - A Case Report and Review of Literature." International Journal of Dental Sciences and Research, vol. 2, no. 5 (2014): 106-108. doi: 10.12691/ijdsr-2-5-1.

\section{Introduction}

Squamous cell carcinoma is the most prevalent malignant neoplasm of the oral cavity. In developing countries, carcinoma of oral cavity in males is the sixth most common cancer after lung, prostrate, colorectal, stomach and bladder cancer. In females, it is the tenth most common site of cancer after breast, colorectal, lung, stomach, uterus, cervix, ovary, bladder and liver [1].

The risk factors for OSCC are tobacco, betel quid, alcohol and recently human papilloma virus infection. The important factors related to carcinoma with a poor prognosis include large size of the tumor at the time of diagnosis, the presence of metastases in regional lymph nodes and a deep invasive front of the tumor.

Presented here is a case of a 62-year-old male patient who reported to our dental OP with a chief complaint of growth on palate since one month.

\section{Case Report}

A 62-year-old male patient reported to our dental OPD with a chief complaint of growth on palate since one month.

Patient was apparently normal one month back. Later he developed a growth which was gradual in onset, initially small in size and gradually progressed to attain the present size. He also gave a history of burning sensation on taking spicy food. No history of associated pain.

Patient gave a history of hypertension since two years and was under medication. Medical history revealed that cataract surgery was done 3 years back. History of betel quid chewing since 35 years with a frequency of 5 times per day and quid it on right buccal vestibule.

On intraoral examination, on inspection, a single ulceroproliferative growth was seen on right posterior slope of hard palate. It was roughly oval in shape and measured approximately 6 X $4 \mathrm{~cm}$ in size. Mediolaterally it extends from midpalatine raphae to marginal gingiva of 16, 17 laterally. Extends from 14 region anteriorly to 18 region posteriorly. Edges were everted. Floor was covered by pseudomembranous slough. The surrounding mucosa appears normal.

On palpation, inspectory findings with respect to site, size, shape and extent were confirmed. Margins were irregular and non-tender on palpation. Bleeding on palpation was also evident. The growth was sessile in relation with deeper structures.

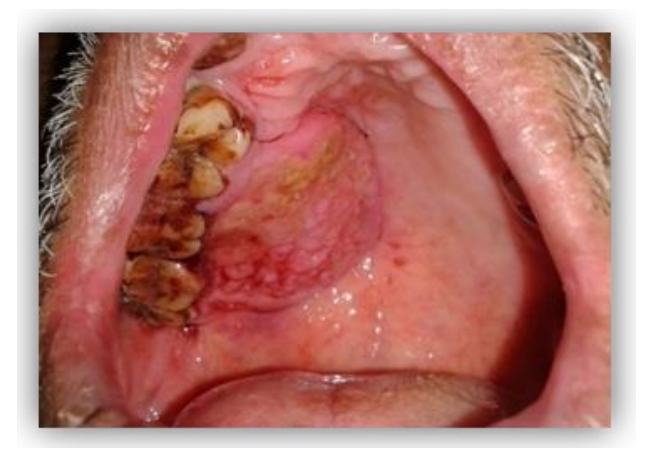

Figure 1. A single ulceroproliferative growth was seen on right posterior slope of hard palate

The positive findings of old age, male patient, ulceroproliferative lesion on palate since one month were 
considered a provisional diagnosis of carcinoma of palate was made.

Differential diagnosis of carcinoma of maxillary sinus, mucoepidermoid carcinoma, acinic cell carcinoma and adenoid cystic carcinoma was considered.

Computed tomography (CT) in axial section, [Figure 2] soft tissue window setting revealed a mass appears to be arising from hard palate. No bony erosion is evident. Ethmoid air cell shows mucosal thickening. Bilateral maxillary sinus shows mucosal thickening. These features are suggestive of a polypoid growth in right maxillary sinus.

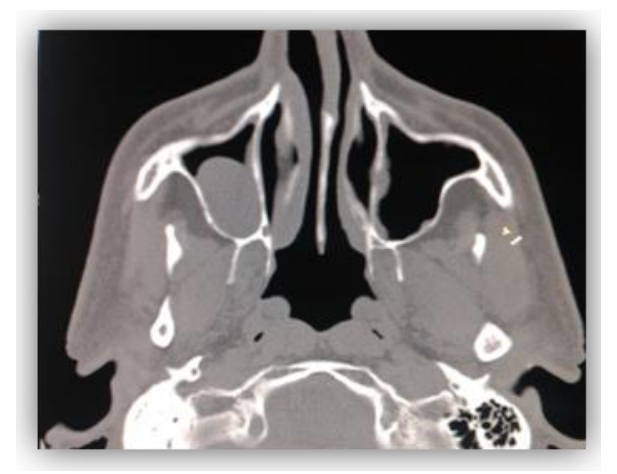

Figure 2. CT scan reveals polypoid growth in right maxillary sinus

An excisional biopsy was done. Histopathology of given section showed multiple bits of tissue exhibiting parakeratinized stratified squamous epithelium and stromal tissue. The epithelium is hyperplastic in many areas and dysplastic in few areas. Few areas show microinvasion into the underlying stroma. Few bits show stromal tissue exhibiting strands of highly dysplastic epithelium showing atypia well formed keratin pearl and areas of necrosis are noted. Dense inflammatory cell infiltrate throughout lesional area. The histopathological section was suggestive of well-differentiated squamous cell carcinoma [Figure 3].

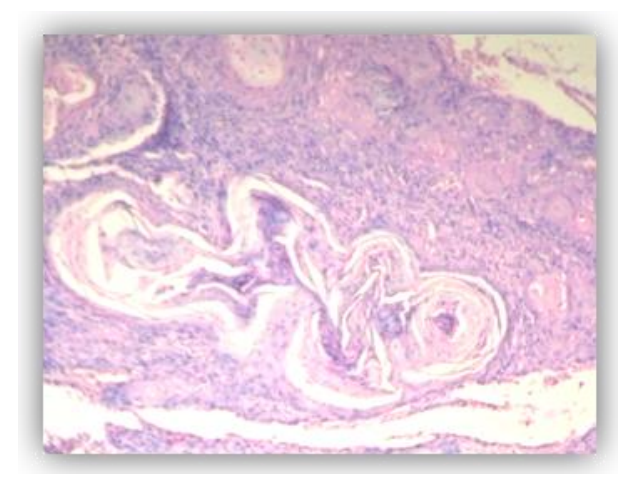

Figure 3. Histopathologic section shows epithelium is hyperplastic in many areas and dysplastic in few areas

\section{Discussion}

OSCC (OSCC) - a disease found particularly in low income communities and mainly a problem of older men.

More than $90 \%$ of all oral cancers are squamous cell carcinoma (SCC) [2].

Lifestyle factors, especially tobacco and alcohol, appear particularly important but, in some cases, betel quid, sunlight exposure, ionising radiation, human papillomavirus
(HPV), genetic or other infections or immuno-incompetence are relevant.

OSCC can arise from a previously existing potentially malignant disorder such as oral leukoplakia, erythroplakia, submucous fibrosis and lichenoid dysplastic lesions, or can arise de novo [3].

The most common site for intraoral carcinoma is the lateral border, posterior and ventral surfaces of the tongue followed by floor of the mouth. Less common sites are the gingiva, buccal mucosa, labial mucosa and hard palate. [4] The case reported here is carcinoma on the palate

The lateral tongue and floor of mouth combine to form a horseshoe shaped of high risk region. There are two main reasons for the high risk region: first, carcinogens mix with saliva, pool in the bottom of the mouth and; secondly, less protection against carcinogens as these regions of oral cavity are covered by a thin, nonkeratinized mucosa [5].

According to Pindborg, OSCCs are classified into histopathologic grades as well differentiated (grade 1), moderately differentiated (grade 2) and poorly differentiated (grade 3). Well and moderately differentiated tumors can be grouped together as low grade and poorly differentiated and undifferentiated tumors as high grade. [6] In this patient, it was a case of well-differentiated squamous cell carcinoma.

The treatment of OSCC generally requires the services of a multidisciplinary team, the main aim of treatment is to eradicate the cancer, to prevent recurrence and finally restore the form and function of the affected parts. Surgery is the preferred first line treatment of small, accessible OSCCs. However, advanced-stage OSCC is usually treated by a combined treatment program of surgery, chemotherapy, and radiotherapy [7]. In cases of recurrent OSCC, EGF-R inhibitor coupled with chemoradiotherapy, is the first line of treatment [8].

Surgical resection of oral carcinoma with tumor free margins of less than $5 \mathrm{~mm}$ may be followed by local recurrence and possibly by distant metastasis, and usually necessitates the administration of post-surgery chemoradiotherapy.

Twenty to thirty percent of cases of resection of OSCC with adequate, wider than $5 \mathrm{~mm}$, tumor-free margins as evidenced on histopathological examination will develop local or contiguous regional "recurrence" [9]. There are two possible explanations for this high- rate of recurrence. First, some carcinomatous keratinocytes may have remained in the margins of the surgical wound, but because there were so few, they were not detected by histopathological examination. Second, the large field of precancerized epithelium comprising pre- cancerous keratinocytes at different stages of transforma- tion from which the primary carcinoma developed, was not removed at the surgical procedure. Epithelium from a field of precancerization may appear normal microsco- pically, or it may be dysplastic. It may also appear nor- mal microscopically, but nevertheless may harbour keratinocytes with cytogenetic alterations including loss of heterozygosity and p53 mutations, or epigenetic changes in methylations of certain promoters of tumor- suppressor genes and DNA repair genes [10]. Following acquisition of additional genetic alterations, either keratinocytes in the dysplastic epithelium or the genetically transformed keratinocytes may become cancerous giving rise to a new 
field carcinoma close to where the primary carcinoma had been excised, creating an impression of recurrence.

To conclude, the stage of advancement of oral SCC at the time of diagnosis is the most important prognostic factor. Despite advances in various treatment modalities such as chemotherapy, radiotherapy, surgery and gene therapy the five-year survival rate for oral cancer has not improved significantly over the past several decades and it remains at about 50 to 55 percent [7].

\section{References}

[1] Landis SH, Murray T, Bolden S, Wingo PA, Cancer statistics, 1999. CA Cancer J Clin 1999; 49: 8-31.

[2] E. Attar, S. Dey, A. Hablas, I. A. Seifeldin, M. Ramadan, L. S. Rozek and A. S. Soliman, "Head and Neck Cancer in a Developing Country: A Population-Based Perspec-tive Across 8 Years," Oral Oncology, Vol. 46, No. 8, 2010, pp. 591-596.

[3] C. Scully and J. Bagan, "Oral Squamous Cell Carcinoma Overview," Oral Oncology, Vol. 45, No. 4, 2009, pp. 301-308.

[4] Neville BW, Damm DD, Allen CM, et al. Oral \& maxillofacial pathology. 2nd ed. Phila., PA: Saunders; 2002; 337-369.
[5] Jovanovic A, Schulten EA, Kostense PJ, et al. Tobacco and alcohol related to the anatomical site of oral squamous cell carcinoma. J Oral Pathol Med 1993; 22: 459-462.

[6] Pindborg JJ, Reichart PA, Smith CJ, van der Wall I. Histological typing of cancer and precancer of the oral mucosa. 2nd ed. Berlin: Springer Verlag; 1997. p. 10-6.

[7] J. P. Shah and Z. Gil, "Current Concepts in Management of Oral Cancer-Surgery," Oral Oncology, Vol. 45, No. 4, 2009, pp. 394401.

[8] J. H. Lorch, M. R. Posner, L. J. Wirth and R. I. Haddad, "Seeking Alternative Biological Therapies: The Future of Targeted Molecular Treatment," Oral Oncology, Vol. 45, No. 4, 2009, pp. 447-453.

[9] B. J. Braakhuis, M. P. Tabor, C. R. Leemans, I. van der Waal, G. B. Snow and R. H. Brakenhoff, "Second Pri-mary Tumors and Field Cancerization in Oral and Oro-pharyngeal Cancer: Molecular Techniques Provide New Insights and Definitions," Head and Neck, Vol. 24, No. 2, 2002, pp. 198-206.

[10] D. Goldenberg, S. Harden, B. G. Masayesva, P. Ha, N. Benoit, W. H. Westra, W. M. Koch, D. Sidransky and J. A. Califano, "Intraoperative Molecular Margin Analysis in Head and Neck Cancer," Archives of Otolaryngol-ogy_-Head and Neck Surgery, Vol. 130, No. 1, 2004, pp. 39-44.

[11] Silverman S Jr. Demographics and occurrence of oral and pharyngeal cancers. The outcomes, the trends, the challenge. J Am Dent Assoc 2001; 132: 7S-11S. 